\title{
Hypercalcemia and Milk Alkali Syndrome: A Case Report
}

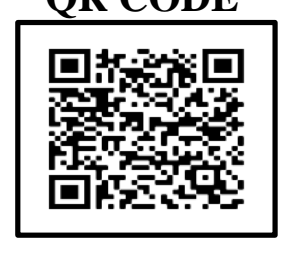

\section{KRITI SETH ${ }^{1}$, NALINI KHARBANDA*2}

A triad of metabolic alkalosis, hypercalcemia and renal insufficiency constitutes the milk alkali syndrome. Elderly subjects, especially those on drugs that GFR are more prone to acquire this syndrome. Those who take calcium supplements have high chances of developing milk alkali syndrome and this stands amongst the top five causes of hypercalcemia. Herein we present a case of hypercalcemia who was taking only small amount of calcium supplements but had a few concomitant risk factors.

KEYWORDS: Hypercalcemia, Milk Alkali Syndrome, Calcium

\section{INTRODUCTION}

Milk-alkali syndrome is characterized by the triad of hypercalcemia, metabolic alkalosis and acute renal failure and is associated with the intake of large amounts of calcium and absorbable alkali. ${ }^{1,2}$ Possible symptoms of hypercalcemia include debility and fatigue, muscle weakness, concentration disorders, nausea, vomiting, anorexia, constipation, polyuria, polydipsia, depressed mood, hypertension, arrhythmia and somnolence. ${ }^{3}$ Aged females taking calcium carbonate supplements for osteoporosis or other reasons are too vulnerable to hypercalcemia. Milk alkali syndrome first was first identified in the beginning of $2 \mathrm{O}^{\text {th }}$ century. With the introduction of $\mathrm{H}_{2}$ blockers and proton pump inhibitors, the incidence of Milk-alkali syndrome decreased, but a resurgence of this syndrome has been witnessed because of the wide availability and increasing use of calcium supplements.

\section{CASE REPORT}

A 79-year-old female patient was referred by her family physician to evaluate her hypercalcemia. On admission, the patient complained of confusion, anorexia, nausea, polyuria, weakness in arms and legs, nausea and alternating stool consistency. Increasing forgetfulness in the past 2 weeks was reported by the accompanying person. Vital signs were in normal range, the patient was well oriented to place and time and no neurological deficits were noticed. Laboratory tests confirmed the presence of hypercalcemia at $2.8 \mathrm{mmol} / \mathrm{L}$. Calcium levels at the family physician's clinic two days before were $3.5 \mathrm{mmol} / \mathrm{L}$. The dose of the diuretic was increased in view of cardiac decompensation a few days before the occurrence of hypercalcemia with a simultaneous increase in calcium supplementation. Other routine blood parameters were notable for increased creatinine at $158 \mathrm{pmol} / \mathrm{L}$. The ECG, chest $\mathrm{X}$-ray and urine examination were all normal. At the time of admission, patient was taking acetylsalicylic acid, lisinopril, furosemide, atorvastatin, calcium carbonate supplements and vitamin D. Parathyroid hormone levels were found to be low, excluding PTH-mediated hypercalcemia as well as primary hyperparathyroidism. Laboratory tests revealed reduced 1.25-OH-Vitamin $\mathrm{D}_{3}$.

Evidence of respiratory alkalosis was found on performing arterial blood gas analysis with a compensatory increase in carbon dioxide partial pressure. Further investigation revealed an increase in the bicarbonate levels. Calcium and vitamin D supplementation were stopped, diuretic therapy was discontinued and forced hydration was started for the treatment of hypercalcemia. Calcium levels the following day to $2.53 \mathrm{mmol} / \mathrm{L}$ and the patient reported a reduction in level of nausea, fatigue, weakness and polyuria. As calcium levels lowered down to normal and remained in range after forced hydration, malignancy or a paraneoplastic cause were excluded. Vitamin D intoxication was also ruled out as 25 -hydroxy vitamin $D_{3}$ levels were in normal range. There was no evidence indicating pathological conditions like granulomatous diseases, sarcoidosis, tuberculosis or lymphomas. Hyperfunctioning thyroid and Adrenocortical insufficiency were also excluded on basis of normal TSH values (along with absent classic hyperthyroidism 
symptoms) and normal morning cortisol levels respectively. The congenital metabolic disorder of familial hypocalciuric hypercalcemia was ruled out on cross confirming the patient's previous checkup reports from family physician. High level of creatinine ( 167 $\mu \mathrm{mol} / \mathrm{L})$ and low glomerular filtration rate (24 $\mathrm{mL} / \mathrm{min} / 1.73 \mathrm{~m}^{2}$ ) suggesting an acute renal failure noted in addition to hypercalcemia and metabolic alkalosis lead to a diagnosis of milk-alkali syndrome.

\section{DISCUSSION}

Increased calcium levels are challenging for physicians and non-specific symptoms are problematic. ${ }^{3}$ The search for a definite diagnosis is essential in view of the diverse treatment options. In hypercalcemic subjects, it is important to confirm the laboratory values with a second blood sample and correcting it against the serum albumin. This helps in ruling out pseudohypercalcemia occurring due to dehydration or other causes and helps in formulation of a correct diagnosis. Increased calcium is confirmed by a second blood sample and PTH determined simultaneously. Past medical history and records are also helpful in assessing the dynamics of the metabolic disorder. Majority of cases of hypercalcemia are due to primary hyperparathyroidism or a (para)neoplastic cause. A detailed drug history and evaluation of $25-\mathrm{OH}$-vitamin $\mathrm{D}_{3}$ and 1,25-OH-vitamin $\mathrm{D}_{3}$, blood gas analysis, $\mathrm{PTHr}$ and thyroid stimulating hormone are helpful in evaluating PTH-independent hypercalcemia.

Milk-alkali syndrome consists of hypercalcemia, various degrees of renal failure, and metabolic alkalosis due to ingestion of large amounts of calcium and absorbable alkali. This syndrome was first identified after medical treatment of peptic ulcer disease with milk and alkali was widely adopted during early zoth century [4]. Other differential diagnostic etiologies for hypercalcemia must also be excluded. When $\mathrm{H}_{2}$ blockers and proton-pump inhibitors were introduced for medical use, there was a decrease in the incidence of milk-alkali syndrome. Milk alkali syndrome is reported to be the third most common cause of hypercalcemia after hyperparathyroidism and malignant neoplasms.5,6 The commonly affected subjects have comorbid conditions or risk factors like elderly women taking calcium supplements for osteoporosis, subjects with chronic renal disease, people at high risk for volume depletion and people who use calcium supplements or antacids at high doses or drugs that may reduce the glomerular filtration rate. ${ }^{3}$
Hypercalcemia causes renal vasoconstriction with reduced GFR. The activation of calcium-sensing receptors in the ascending limb of the loop of Henle slows down sodium-potassium-chloride transporters, resulting in increased natriuresis and diuresis leading to a fluid deficit. Hypercalcemia also slows down ADHdependent water reabsorption, which leads to further volume depletion and further reduces pre-renal glomerular filtration. Intake of absorbable alkalis, impaired renal function, and increased tubular bicarbonate absorption require and maintain metabolic alkalosis, which in turn leads to calcium reabsorption via a $\mathrm{pH}$-sensitive calcium channel in the distal tubule, thereby maintaining hypercalcemia. Evidence based therapy consists of cessation of all calcium- and carbonate-containing or alkaline preparations and definite forced hydration at the start with calcium-free infusion solutions. Immediate administration of calciuric loop diuretics is not recommended since they may result in an electrolyte imbalance, hypovolemia or renal impairment. ${ }^{1}$ The possible use of loop diuretics after rehydration should be assessed clinically based on volume status. Calcium-sparing diuretics are contraindicated. Bisphosphonates should not be used due to the high risk of consequent hypocalcemia with milk-alkali syndrome. Calcium levels returning to normal within a few days and remaining normal also indicates the presence of milk-alkali syndrome.

Evidence in the literature suggests that pure metabolic alkalosis may be absent in cases with pre-existing chronic renal failure. ${ }^{7}$ It was suspected that suspending calcium carbonate intake and reducing diuretics in consultation with the family physician before referral interrupted the vicious circle of hypercalcemia, leading to reduction metabolic alkalosis and regression of hypercalcemia.

\section{CONCLUSION}

The presence of non-specific symptoms such as nausea, fatigue, low threshold serum calcium and albumincorrected calcium levels calculated should direct the healthcare professionals to take hypercalcemia into consideration. Detailed drug intake history and PTH measurement helps in formulating initial diagnosis of hypercalcemia. There are numerous causes of hypercalcemia like multiple myeloma, thyrotoxicosis, primary hyperparathyroidism, malignant neoplastic lesions and calcium or vitamin D intoxication. Milkalkali syndrome should be considered if hypercalcemia, alkalosis and acute renal failure are present. 


\section{REFERENCES}

1. Felsenfeld AJ, Levine BS. Milk alkali syndrome and the dynamics of calcium homeostasis. Clin J Am Soc Nephrol. 2006;1(4):641-54.

2. Medarov BI. Milk-alkali syndrome. Mayo Clin Proc. 2009 Mar;84(3):261-7. doi: 10.1016/Soo25-6196(11)61144o. PMID: 19252114; PMCID: PMC2664604.

3. Kohler S, Schmid C, Krayenbühl PA. Hyperkalzämie. Praxis (Bern 1994). 2009 Mar 4;98(5):233-40. doi: 10.1024/1661-8157.98.5.233.

4. Cope CL. Base changes in the alkalosis produced by the treatment of gastric ulcer with alkalies. Clin Sci. 1936;2:287-300.

5. Beall DP, Scofield RH. Milk-alkali syndrome associated with calcium carbonate consumption: report of 7 patients with parathyroid hormone levelsand an estimate of prevalence among patients hospitalized with hypercalcemia. Medicine (Baltimore). 1995;74(2):89-96.

6. Patel AM, Goldfarb S. Got calcium? Welcome to the calcium-alkali syndrome. J Am Soc Nephrol. 2010 Sep;21(9):1440-3. doi: 10.1681/ASN.2010030255.

7. Kapsner P, Langsdorf L, Marcus R, Kraemer FB, Hoffman AR. Milk-alkali syndrome in patients treated with calcium carbonate after cardiac transplantation. Arch Intern Med. 1986;146(10):1965-8.

Source of support: Nil, Conflict of interest: None declared

Cite this article as:

Seth K, Kharbanda N. Hypercalcemia and Milk Alkali Syndrome: A Case Report Int Healthc Res J. 2020;3(12):380-382. https://doi.org/10.26440/IHRJ/0312.03326 